\title{
The theory of granular packings for fine soils
}

\author{
Calixtro Yanqui \\ San Agustin National University, Arequipa, Peru
}

\begin{abstract}
Fine soils are substances made of tiny particles, for which, besides their geometrical assemblage, the electrochemical forces are important. But the broad experience in Soil Mechanics has shown that, excluding the time, they behave like granular materials. By using the scanning electron microscopy, it has been shown by several authors that clay assemblages are structures of the second order, made of solid particles grouped in clusters. Applying the theory of packings of spheres in contact to the intra-cluster structure as well as to the entire mass of soil, a fundamental equation is obtained. The plastic limit is found to be a constant for a geological formation, because it is obtained by eliminating the capillary water and transforming the clay mass into a first order structure. The liquid limit is a inherently property of the second order, due to the development of clusters. Consequently, there is a linear relationship between the plasticity index and the liquid limit, with a slope of unity, as can be deduced from the abundant data reported by several authors around the world.
\end{abstract}

\section{Introduction}

In spite of its importance, a structural model to describe quantitatively the fine soils behavior has not been developed yet, mainly due to platelet shape of their particles, as well as their complicated assemblage, and the effect of bonding. However, the consolidated and drained shear tests have shown that bonding is a time dependent variable, whose effect becomes null after a long time. In this paper, the theory of granular packings, that has proved to be useful to explain several features of sands, is adapted to take into account the other two features.

\section{Assemblage of fine grains}

Fine soils are made of very small particles. Qualitatively, it is known that, if two clay particles are brought them toward each other, they exert forces on each other; basically, repulsion due to their electrical charge, and attraction, due to the van der Waals or secondary bonding force. According to the predominance of some force, fine soils flocculate or disperse; causing the soil to have very open assemblage. From recent studies on real clay soils using the scanning electron microscopy [1], the individual particles appear to be aggregated or flocculated together in an assembly of small units called domains, which in turn group together to form clusters, that are large enough to be seen by a simple microscope (Fig. 1). Other authors classify the fabric of clays in two classes: elementary particle arrangements, and groups of clay platelets [2]. Powers [3] arrives to the same conclusion, when studying the structure of the fresh cement paste, defining two kinds of evaporable water: the adsorbed water, held by the individual particles by short-range forces, and the capillary water, filling the much larger pores. As early as 1962, Olsen [4] showed that, for explaining the hydraulic conductivity, finegrained soils should be modeled as clusters of spherical shape.

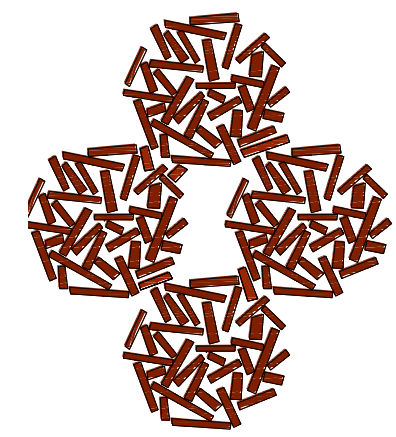

Fig. 1. Clay structure, modified from Collins and McGown [1]

\section{Theory of granular packings}

A grain may be defined by two diameters: the equivalent diameter, $D$, and the diameter of contact between two grains, $D_{\text {c. }}$. So that, a set of grains can be assembled in packings of equivalent spherical grains, whose centers are at the points of the crystallographic Bravais lattices whose edges are of the same length, and equal to the diameter of contact between the spheres, $D_{\text {c }}$ (Fig. 2): the trigonal bipyramid and the rhombohedron. According to the principle of multiplicity, the spatial orientation adopted by this rhombohedron depends on the cinematic conditions imposed by the applied stress.

\footnotetext{
* Corresponding author: cyanquimurillo@,hotmail.com
} 


\subsection{Phase relationships}

In general, it is always possible to choose a parallelepiped as a primitive cell whose vertices are the centers of eight neighboring spheres, not necessarily all in contact. Now, the total volume of this cell is $V=a b c \operatorname{sinacos} \zeta$, and the volume of the grain, $V_{\mathrm{s}}=(\pi / 6) D^{3}$; (Fig. 2.a). Thus, the voluminosity of the parallelepiped can be found from the expression [5]:

$$
v=\frac{6 a b c}{\pi D^{3}} \sin \alpha \cos \varsigma
$$

where $u$ stands for the voluminosity of the packing of spheres; $a, b$, and $c$, are the lengths of the edges of the parallelepiped; $a$, the acute angle forming by the two edges of the horizontal face; and $\varsigma$, the azimuth angle of the non horizontal edge. It is worth to mention at this point that there are sophisticated models to describe some additional features of granular packings, such as the grain shape and the polidispersivity [6] [7] [8].
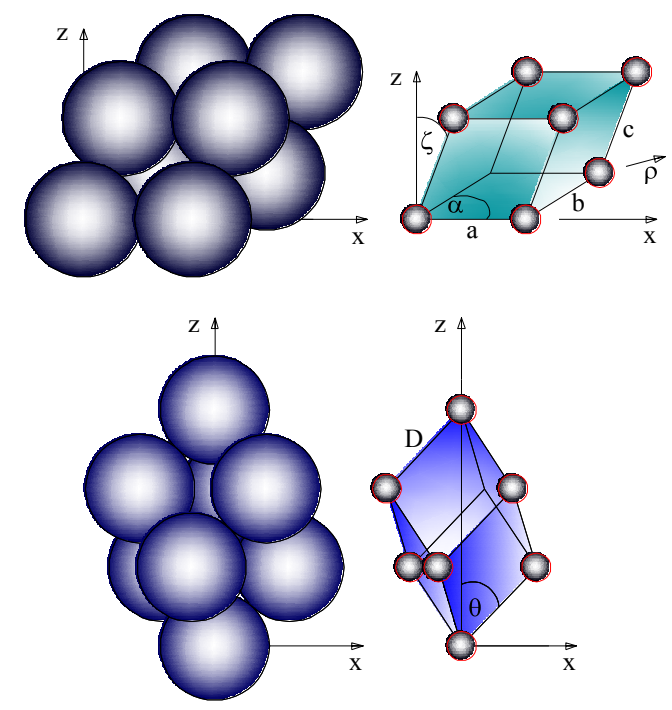

a)

b)

Fig. 2. a) For $a \neq b \neq c$, the general packing and its associated crystallographic cell, and specially, for $a=b=c=D_{c}$, the distortional rhombohedron, $b$ ) the elongational rhombohedron

\subsection{Mechanically allowable packings}

In engineering, the final object of the packings is the study of the stress-strain relationship of the granular matter. For it, packings are to be cinematic and statically admissible. The first condition ensures the continuous deformation of the mass, and the second, the stress transfer form one grain to another. Consequently, from the fourteen Bravais cells, only the monoclinic, the rhombohedric and the bipyramidal cells are acceptable. But, only the rhombohedron allows the configuration of chains of contact forces, and the development of large deformations, regarding the experimental behavior of soil. However, the cell of this packing must not necessarily match the basic parallelepiped used to calculate the phase relationship.

Distortional kinematics of a packing requires that one of the faces of the rhombohedron be horizontal and the principal diagonal be on the plane of mirror symmetry (Fig. 2.a). Under these conditions, the azimuth angle becomes the shear angle. So that $\varsigma=\beta$. Besides, the maximum deformation capacity is achieved when $\alpha=90^{\circ}$, and the wedges of the parallelepiped becomes $a=b=c=D_{c}$. Then, defining the textural parameter $\mathrm{X}=\left(D_{\mathrm{c}} / D\right)^{3}$, equation $(1)$ may be specialized to:

$$
v=\frac{6}{\pi} \chi \cos \beta
$$

For elongational kinematics of a packing, the principal diagonal of the rhombohedron is vertical, and the lines of contact are axisymmetric with respect to this axis, according to the angle $\theta$ (Fig.2.b). Then, $\varsigma=\theta$. These conditions are only possible if the base of the basic parallelepiped is made of two equilateral triangles, resulting in $\mathrm{a}=60^{\circ} ; a=b=D_{\mathrm{c}} \sqrt{3} \operatorname{sen} \theta$, and $c=D_{\mathrm{c}}$. Then,

$$
v=\frac{9 \sqrt{3}}{\pi} \chi \sin ^{2} \theta \cos \theta
$$

\subsection{Packings duality}

Analysis of equations (2) and (3) shows that, for two physical values of the angle of distribution, there is a single value of the voluminosity. This property has been called duality of packings and it is one of the fundamental concepts in interpreting the behavior of soils. Indeed, these functions show two branches, separated by a peak value: the first associated with the increasing of the voluminosity, describing a dilative state, and the second, related to the decreasing of the voluminosity, revealing a contractive state.

\subsection{Extreme states}

In addition, due to its discrete nature, the angles involved in these functions have physical meaning in a limited range, whose boundaries are associated with the densest states, which are achieved when the number of contacts between spheres is increased. For a distortional packing, the loosest state is attained when $\beta=0^{\circ}$, and the voluminosity is 1.91; and the two densest states are obtained when $\beta=45^{\circ}$ and $\beta=-45^{\circ}$, for which the voluminosities coincide with each other and are 1.35 , In terms of the volume fraction, the extreme values are 0.523 and 0.74 , respectively. For an elongational packing, the loosest state is found when $\cos \theta=1 / \sqrt{ } 3$, being the voluminosity 1.91 ; the densest state for a dilative packing is attained when $\sin \theta=1 / \sqrt{3}$; being the voluminosity 1.35; and the densest state for the contractive packing is obtained when $\cos \theta=1 / 3$, being the voluminosity 1.47 . So that, the extreme values of the volume fraction are $0.523,0.74$ and 0.68 , respectively.

\subsection{Submerged ordered packings}

The special behavior of the submerged fine solid particles can be described by the Gouy-Chapman theory of the diffuse double layer. Accordingly, a water layer of 
very small thickness, $a_{\mathrm{p}}$, is adsorbed onto the solid particle due to chemical interactions and Coulomb electrical attraction, increasing the diameter of contact to: $D_{\mathrm{p}}=D+2 a_{\mathrm{p}}$, (Fig. 3). This layer displays colloidal properties, and, consequently, originates the plasticity of fine soils. The voluminosity of the elongational packing of these bigger spheres may be found as:

$$
v_{w}=\frac{9 \sqrt{3} \chi}{\pi}\left(1+\frac{2 a_{p}}{D}\right)^{3} \sin ^{2} \theta \cos \theta
$$

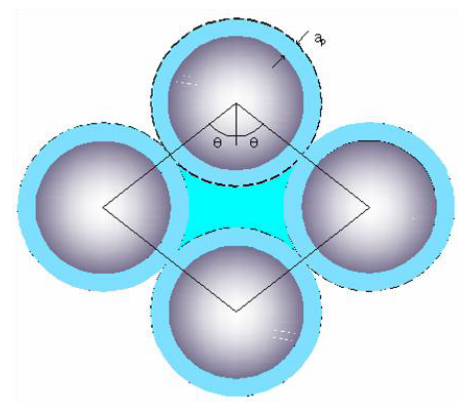

Fig. 3. Two dimensional packing of submerged tiny spheres

\section{Structure of fine soils}

Since the beginning of the Soil Mechanics, the authors recognize that structure of clays is related to the assemblage of clusters. This means that fine soils may modeled as a packing of packings, as a second order structure (Fig. 4). Within the cluster solid particles are modeled as spheres of contact assembled in a first order packing, whereas, all the soil mass is modeled as a second order packing of spherical clusters of contact. Hence, by definition, the voluminosity of the fine soil is found as $\mathrm{U}=V / V_{s}$. If $V c$ is the volume of the cluster, this relationship can be written as: $\mathrm{U}=\left(V_{\mathrm{d}} / V_{s}\right)\left(V / V_{\mathrm{c}}\right)$, or:

$$
v=v_{1} v_{2}
$$

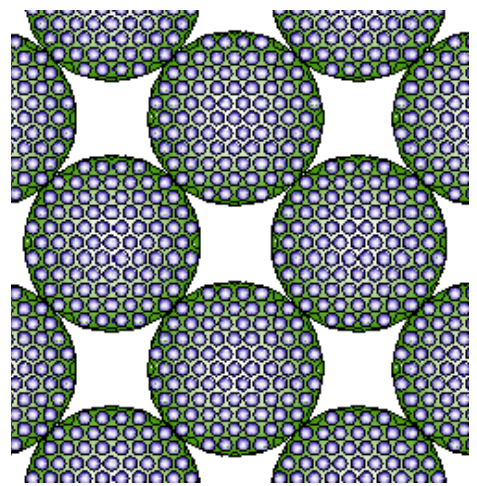

Fig. 4. Two dimensional packing of spherical clusters, which at the same time, are packings of spherical particles

where $v_{1}$ is the first order voluminosity, and $u$, is the second order voluminosity. In general, $v$ is related to the saturated water content, $w$, by the equation $\cup=G w+1$, being $G$ the specific gravity of the solid particles. In terms of this quantity, $\mathrm{U}_{1}$ is associated to the adsorbed water, whereas $\boldsymbol{u}$, to the capillary water. Equation (5) is the fundamental equation to be used in analyzing the plasticity parameters standardized by the Soil Mechanics, and the Granular Mechanics of fine soils. The plastic limit is obtained by distorting a clay ball exerting some kind of compression to transform it into a cylinder by rolling it several times until fissures. This phenomenon occurs when the capillary water is totally eliminated, and the soil assemblage becomes a first order alone. Consequently, the plastic limit stands for the densest state of plastic soils, and depends only on the properties of the particles, for what it remains constant for a geological formation. The liquid limit, which is obtained by hitting a loose sample on a flat surface, is supposed to stands for the densest state of the second order structure. Numerically, if there is not capillary water, the soil is at the plastic limit, $\mathrm{U}_{2}=1$, and $\mathrm{u}=\mathrm{U}_{1}=$ $1.35 \mathrm{X}=$ constant; if the soil is at the liquid limit, $\mathrm{v}_{1}=1$, and $u=u_{2}$, that depends on the sample of the geological formation.

\section{Validation of results}

The difference between these two limits is called plasticity index and is denoted by $I_{\mathrm{p}}$.

$$
I_{p}=w_{l}-w_{p}
$$

As the plastic limit is a constant, this quantity varies linearly with the liquid limit. It is worth to mention that this observation was already known by the pioneers of the soil mechanics. The contribution of the theory of granular packings is the establishment of the slope of the line as the unity, if the soil comes from the same geological formation.

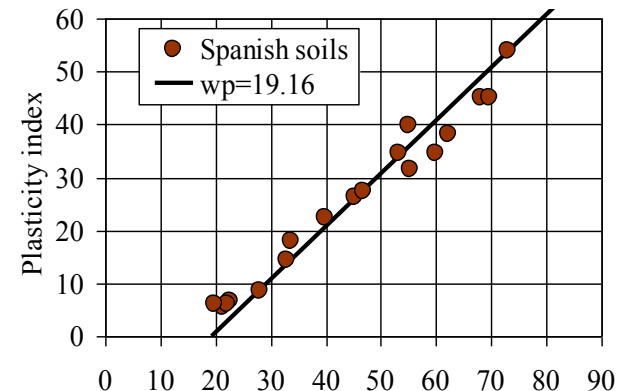

a)

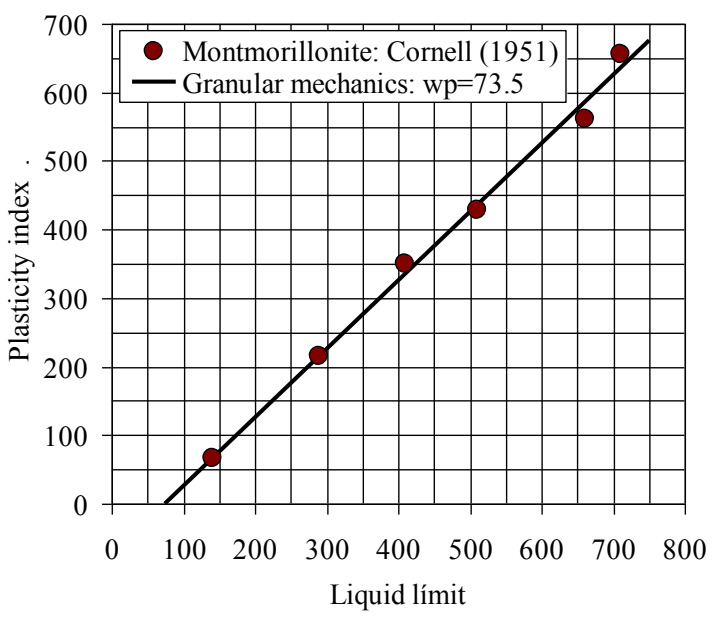

b) 

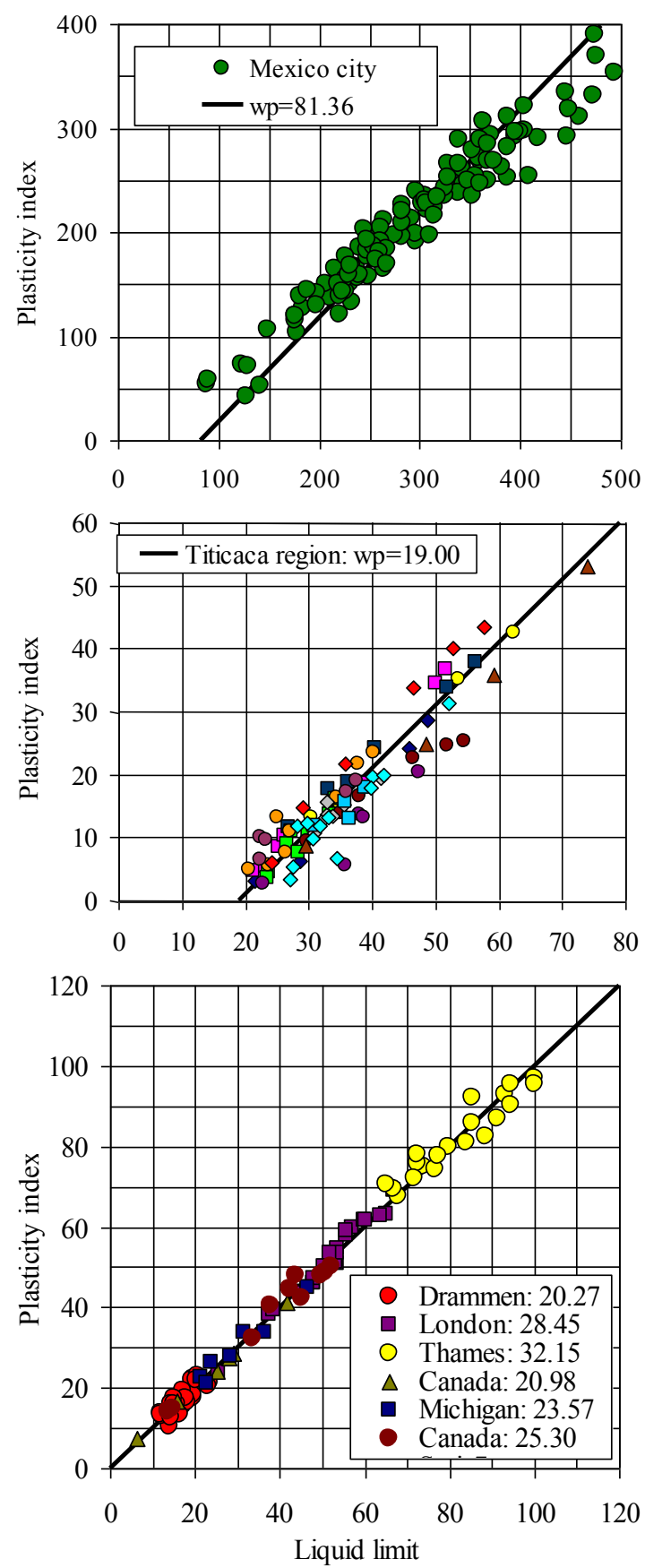

Fig. 5. Relationship between the liquid limit and the plasticity index for: a) Spanish soils [9] b) montmorillonite [10], c) Mexico city clay [11], d) Southern Peru clays, e) several clays around the world, compiled by Lambe and Whitman [12]

Geologically, these findings may be interpreted as follows; the plastic limit defines the nature of the fine particles, and the liquid limit stands for the effect of some other geological feature. As a matter of fact, the experimental data of some clay formations from different places of the world, usually obtained during normal subsoil prospecting work, and reported by various authors, fit very well with equation (6), where $w_{\mathrm{p}}$ is the average of all site plastic limits. Besides, it is interesting to notice that the experimental plasticity index never is zero, revealing the jump from the plastic limit to the liquid limit, associated to the development of clusters or, on the opposite way, capillary water voids.

\section{Conclusions}

Fine soils are made of assemblages of clusters of tiny particles, displaying structures of the second order. In spite of this complication, fine soils can be described using the theory of granular packings. Within the cluster, the packing is the same as the one of coarse grains. The entire soil mass may be considered as a packing of clusters, modeling the structure of the second order. The plastic limit is obtained in such a way that the capillary water disappears, becoming a property of the clay composition and, therefore, a constant for the geological formation. The liquid limit is obtained in loose samples. So, it stands for the second-order structure of fine soils. As long as the plastic limit is a constant, the plasticity index is linearly related to the liquid limit, with a slope of unity. The data reported by several authors confirm this finding.

\section{References}

1. R.N. Yong, D.E. Sheeran, Fabric unit interaction and soil behaviour. Proc. Symp. On Soil Structure, Sweden, 176-183 (1973)

2. K. Collins, A. McGown, The form and function of microfabric features in a variety of natural soils. Geotechnique XXIV, 223-254, (1974)

3. T.C. Powers, The properties of fresh concrete. John Wiley. New York. 1968.

4. H.W. Olsen, Hydraulic flow throw saturated clay. Proceedings Ninth Nat. Conf. on Clays and clay minerals, 131-161 (1962)

5. C. Yanqui, The theory of packings for coarse soils. AIP Conf. Proc. 1542, 341-344 (2013)

6. T. Aste, Circle, sphere, and drop packings. Physical Review E, Vol. 3, No. 3, 2571-2579. (1996)

7. F. de Larrard, T. Sedran. Optimization of ultra high performance concrete by the use of packing model. Laboratorie Central des Ponts and Chaussées (1996)

8. G. Mollon, J.D. Shao, Fourier-Voronoi based generation of realistic samples for discrete modelling of granular materials. Granular Matter. 14(5), 621-638 (2012).

9. J.A. Jimenez, J.L de Justo, Geotecnia y cimientos, Tomo I. Editorial Rueda. Madrid (1975)

10. Cornell University, Final report on soil solidification research. New York (1951)

11. R.J. Marsal, Unconfined compression and vane shear tests in volcanic lacustrine clays. Proc. ASTM Conf. on Soils for Eng. Purposes, Mexico (1959)

12. T.W. Lambe, R.V. Whitman, Soil Mechanics. John Wiley and Sons. New York, (1969). 